\title{
Reflexiones generales sobre el Sistema Nacional de Archivos de la República de Cuba desde la perspectiva del modelo de la continuidad de los documentos
}

\author{
Jorge del Castillo Guevara*
}

Sarah Paz Martín*

Artículo recibido:

27 de septiembre de 2018

Artículo aceptado:

4 de marzo de 2019

Artículo de revisión

\section{Resumen}

Cuba se encuentra en un proceso de reestructuración de su modelo económico y social de desarrollo. El Sistema Nacional de Archivos de la República de Cuba debe desempeñar un rol protagónico en este sentido; sin embargo, su modelo de gestión, basado en los enfoques teóricos más tradicionales, dificulta que pueda responder de manera efectiva ante las demandas y requisitos de la sociedad cubana contemporánea. En este artículo se realizó un análisis sobre dicho sistema, desde los enfoques del modelo australiano de la continuidad de los documentos y tres de sus principios enunciados por Frank Upward. Se utilizó como

\footnotetext{
Facultad de Comunicación de la Universidad de La Habana, Cuba

guevara@fcom.uh.cu sarah.paz@fcom.uh.cu

INVESTIGACIÓN BIBLIOTECOLÓGICA, vol. 33, núm. 81, octubre/diciembre, 2019, México, ISSN: 2448-8321 pp. 89-110
} 
método de investigación el análisis documental. Ello facilitó una mejor comprensión sobre el pensamiento del continuo australiano, así como la identificación de aquellas cualidades que se reconocen como fortalezas para cualquier sistema archivístico en la era poscustodial. Además, se revisaron las principales herramientas jurídicas del Sistema Nacional de Archivos cubano, lo cual permitió examinar algunos aspectos que reflejan su marcado enfoque custodial y patrimonial.

Palabras clave: Modelo de la Continuidad de los Documentos; Paradigma Poscustodial; Sistema Nacional de Archivos; Cuba

General reflections on the National Archives System of the Republic of Cuba from the perspective of the Records Continuum Model

Jorge del Castillo Guevara and Sarab Paz Martín

\section{Abstract}

Cuba is in the process of restructuring its economic and social development model. The National Archives System of the Republic of Cuba must play a leading role in this regard. However, its management model, based on the most traditional theoretical approaches, makes it difficult to respond effectively to the demands and requirements of contemporary Cuban society. This article analyzed such system, based upon the Australian continuum model of documents and three of its principles enunciated by Frank Upward. Documentary analysis was used as a research method and this facilitated a better comprehension of the Australian thinking continuum, as well as the identification of those qualities recognized as strengths for any archival system in the postcustodial era. In addition, the main legal and procedural tools of the Cuban National Archive System were reviewed, which allowed examination of some aspects that reflected its marked custodial and patrimonial approach.

Keywords: Records Continuum Model; Postcustodial Paradigm; National System of Archives; Cuba 


\section{INTRODUCCIÓN}

—1 1 Sistema Nacional de Archivos de la República de Cuba (SNARC) se deEvela en un entorno social, político, administrativo y tecnológico de creciente complejidad, como resultado de los cambios en las estructuras organizacionales, los emergentes modos de gestión y la penetración cada vez más evidente de las tecnologías de información y comunicación en la vida de los individuos, las organizaciones y la sociedad en general. Tales cambios se dan en el marco de lo que se ha llamado "actualización" del modelo económico y social $^{1}$ y de un proceso de informatización de la sociedad cubana. ${ }^{2}$

Todo ello ha provocado un ingente aumento de la producción de documentos e información en diferentes formatos en la administración pública y en el sector empresarial, como resultado de sus actos y transacciones de negocios. Tales documentos e información reflejan una parte importante de la memoria de la sociedad cubana, pues dan fe de la manera en que el gobierno y la administración realizan su gestión y se relacionan con los ciudadanos.

En este contexto, se hace necesario contar con sistemas archivísticos que tengan la capacidad de responder, de manera efectiva, ante esa realidad compleja, que se involucren en los procesos gerenciales desde una perspectiva integradora e inclusiva. Estos sistemas tienen una insoslayable responsabilidad en la articulación de herramientas y procesos que propicien los mecanismos para aspectos que hoy se consideran cruciales como la rendición de cuentas, la transparencia y la responsabilidad pública, sobre la base de un entorno de relaciones confiable.

Hace más de una década, Michael Piggott y Sue McKemmish se cuestionaron qué debía esperar la sociedad de los archiveros. Sobre la base de esa interrogante se adentraron en un debate en torno a la misión de los archiveros, según la Australian Society of Archivist, en lo que respecta al mantenimiento y uso de los documentos como evidencia auténtica de las actuaciones en los planos corporativo, cultural e intelectual, y su papel en la eficacia y la responsabilidad organizacional. En tal sentido, consideraron el modelo australiano de la continuidad como un marco amplio, inclusivo y complejo de transaccionalidad y contextualidad, con potencialidades para la construcción de la memoria

1 Este proceso de los últimos 10 años se ha caracterizado por un grupo de transformaciones en el orden económico y social del país. Según el documento aprobado en el VII Congreso del Partido Comunista de Cuba, el objetivo estratégico del Modelo de Desarrollo es "impulsar y consolidar la construcción de una sociedad socialista próspera y sostenible en lo económico, social y medioambiental, comprometido con el fortalecimiento de los valores éticos, culturales y políticos forjados por la Revolución, en un país soberano, independiente, socialista, democrático, próspero y sostenible" (Partido Comunista de Cuba, 2016: 10).

2 La Política Integral para el Perfeccionamiento de la Informatización de la Sociedad en Cuba fue aprobada en julio de 2017. 
social por medio de procesos de pluralización y acceso de la información, que supera los sesgos de los tradicionales enfoques archivísticos (Piggott y McKemmish, 2002).

El presente artículo tuvo como objetivo analizar el SNARC desde los enfoques del modelo australiano de la continuidad de los documentos, específicamente a partir de tres principios enunciados por Frank Upward en el primero de dos artículos emblemáticos, titulado "Structuring the records continuum - part one: Postcustodial principles and properties" y publicado en la revista Archives and Manuscripts, en 1996.

Con ese fin se realizó un análisis documental de la literatura recopilada sobre los enfoques más contemporáneos de la archivística, en especial aquella que hace referencia al modelo australiano. Ello resultó de gran utilidad para comprender los principios sobre los cuales se basó la reflexión en torno al caso cubano. Luego, se examinaron las regulaciones y procedimientos del SNARC, en especial el Decreto Ley (en adelante, DL) 265/09 "Del Sistema Nacional de Archivos de la República de Cuba", la Resolución 41/09 "Lineamientos para la Conservación de las Fuentes Documentales", y el Manual de procedimientos para el tratamiento documental (Marchena et al., 2008).

También se revisaron otras normativas como el Reglamento de la Ley 107/11 "De la Contraloría General de la República de Cuba", la Resolución 60/11 "Sobre las Normas del Sistema de Control Interno", el DL 281/11 "Del Sistema de Información del Gobierno", el DL 281/07 "Reglamento para la Implantación y Consolidación del Sistema de Dirección y Gestión Empresarial Estatal" y el DL 199/99 "Sobre la Seguridad y Protección de la Información Oficial".

El análisis de este marco regulatorio, el conocimiento empírico de los autores sobre el funcionamiento del SNARC y el diálogo crítico con los enfoques de modelo australiano de la continuidad de los documentos permitieron arribar a los resultados que se ofrecen.

\section{LA ARCHIVÍ́STICA EN EL PARADIGMA POSCUSTODIAL}

Desde la década de 1980 se desarrolló un proceso revolucionario en la archivística si se analiza desde la perspectiva paradigmática de Thomas Samuel Kuhn, quien a partir de su controversial obra The estructure of scientific revolutions presentó una visión sobre el desarrollo de la ciencia contraria a la concepción acumulativa y lineal del conocimiento científico. En este sentido, defendió que las ciencias no evolucionan a partir de la acumulación de conocimiento, sino a partir de revoluciones científicas. Las revoluciones científicas 
se consideran "episodios de desarrollo no acumulativo en que un antiguo paradigma es remplazado, completamente o en parte, por otro nuevo e incompatible" (Kuhn, 1971: 149). El uso de esta metáfora ocurre ante la crisis que provoca la insuficiencia del paradigma anterior.

Sobre la base de las ideas kuhnianas ha tenido lugar un extenso debate en torno a la archivística. No han sido pocos los acercamientos a ese enfoque de desarrollo evolutivo y discontinuo de la ciencia y sus paradigmas para analizar el devenir de esta área del conocimiento, como se puede constatar en la producción científica a partir de la década de 1980.

Silva y Ribeiro (2010) identificaron dos momentos paradigmáticos:

1. Un paradigma custodial, patrimonialista, historicista y tecnicista de 1898 hasta la década de 1980, con la presencia de un enfoque historicista y patrimonialista sobre el alcance de los archivos históricos y administrativos, la gestión de documentos, la normalización y una reformulación crítica de la noción de fondo, entre otros aspectos.

2. Un paradigma poscustodial, informacional y científico desde la década de 1980 hasta la actualidad, en la cual los archivos son tratados como sistemas de información y hay un redimensionamiento epistemológico de la archivística en el campo de la ciencia de la información.

Por su parte, Fernanda Ribeiro se refiere a la presencia de un paradigma poscustodial como resultado de la crisis manifiesta del paradigma tradicional ante las condicionantes sociales, económicas, culturales y tecnológicas de la segunda mitad del siglo XX que "cuestionaron sus fundamentos esenciales y generaron las condiciones para que comenzara a surgir y afirmarse de manera irreversible un nuevo paradigma en el campo de la información” (Ribeiro, 2017: 28).

En el centro de los enfoques poscustodiales se encuentra la teoría australiana, en especial el Records Continuum Model, que en su traducción al español se puede entender como modelo de la continuidad de los documentos (MCD), el cual ha ofrecido una perspectiva consistente ante la complejidad de gestionar información archivística en el escenario tecnológico emergente.

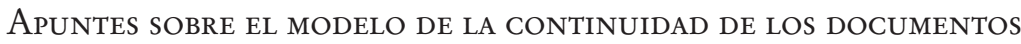 (RECORDS CONTINUUM MODEL)}

Las insuficiencias del enfoque más tradicional del ciclo de vida, centrado en el documento como entidad física, sometido a acciones que ocurren en etapas fijas, circunscrito a roles y estrategias de custodia, con una marcada distinción 
entre records y archives (McKemmish, 1997), condujeron a la rearticulación, por parte de Frank Upward y sus colegas de la Universidad de Monash en 1990, de un enfoque cuyos antecedentes datan de la década de 1950, en la otrora Commonwealth Archives Office (Cumming, 2010): el MCD.

Uno de los principales fundamentos del continuo ha sido el sistema de series australiano, concebido por el archivero y lingüista australiano Peter J. Scott con sus colegas de la Commonwealt Archives Office en la década de 1960 por la complejidad estructural y funcional de la administración australiana y las dificultades para lidiar, de manera efectiva, con la documentación archivística corriente bajo los enfoques teóricos norteamericanos.

Este sistema se sustentó en una interpretación sobre la procedencia, por parte de Scott, diferente a la mirada tradicional. En tal sentido, la procedencia se comprendería a partir de la red de relaciones dinámicas que los documentos presentan con sus múltiples productores y contextos. Así, las entidades -creadores, funciones y documentos- se describían y se relacionaban en diferentes niveles de granularidad.

Los archiveros australianos habían comprendido que supeditar la procedencia a una relación de uno a uno entre los documentos y el productor era una visión reducida y segmentada sobre este principio y no reflejaba su verdadera naturaleza multicontextual. Ahí radica su principal fortaleza y objetividad, por lo que su capacidad de pensar la gestión documental más allá de la disposición física de los documentos, o sea, a partir de las relaciones sincrónicas y diacrónicas entre estos y sus contextos de creación, ha legitimado su aplicación no sólo en el contexto australiano (Cunningham, 2012).

La vigencia del sistema de series se constata hoy en las tendencias actuales de representación de la información archivística en el contexto internacional. Por tanto, no resulta casual que toda la teoría australiana, como asevera Reed (2012), haya estado influenciada por ese enfoque y ha sido el fundamento del pensamiento archivístico australiano.

El MCD fue estructurado en cuatro dimensiones que representan la manera en que se manifiesta el universo archivístico (Upward, 1996; 1997):

- Creación: considerado un sistema de precomunicación, se refiere a los actos realizados por los actores que generan trazas que representan esos actos. En esta dimensión comienza una propagación de los contextos inmediatos de la creación.

- Captura: entendido como un sistema de poscomunicación, la traza se captura en forma de evidencia registrada (records) y se considera evidencia de las actividades de una entidad (organización o individuo). Esto implica una desanidación de los contextos inmediatos de su creación. 
- Organización: la evidencia registrada (records) se organiza como memoria de las funciones de una organización o individuo en forma de archivo, lo que implica una desanidación más profunda de espacio-tiempo.

- Pluralización: la dimensión de mayor distanciación a partir de la diseminación de la memoria colectiva.

También se distinguen cuatro ejes que dividen las dimensiones del modelo:

- Transaccionalidad: se refiere a los documentos (records) como subproductos de las actividades y a los enlaces que estas actividades establecen entre los documentos.

- Identidad: asociado a los actores, productores de documentos, sus particularidades, poder e identidad en perspectivas socioculturales más amplias.

- Evidencialidad: se presentan los documentos como evidencia de los actos que pasan a ser memoria corporativa y colectiva.

- Gestión de documentos: se relaciona con los medios que se utilizan para registrar y almacenar información.

En la Figura 1 se presenta el modelo como ha sido concebido por Upward (1996).

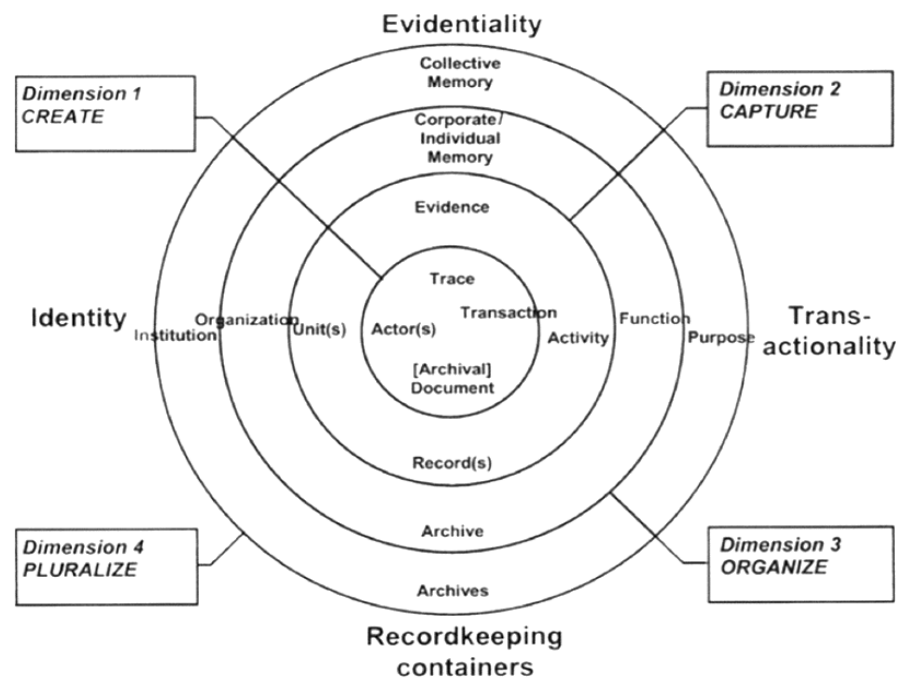

Figura 1. Esquema del records continuum mode/ de Upward (1996) 
Ahora bien, las dimensiones que presenta no deben ser comprendidas desde una perspectiva lineal de ocurrencias secuenciales como en el ciclo de vida, puesto que éstas se manifiestan de manera recursiva en un ir y venir, en el que procesos como la creación y la captura son puntos dentro de una realidad continua.

No es objetivo de este artículo hacer un análisis profundo sobre este modelo. Sin embargo, se hace necesario apuntar algunas cualidades esenciales, que se pueden resumir de la manera siguiente (Cumming, 2010; Gilliland, 2014; McKemmish, 2017; Upward, 2017; Evans, McKemmish y Rolan, 2017):

- El MCD supera el enfoque segmentado de gestión documental condicionado por el ciclo de vida, el cual establece límites de tiempo en un ciclo de etapas que tienen lugar en una línea secuencial de acontecimientos y responsabilidades en el espacio y el tiempo. En este sentido es un marco en el que la gestión documental se comprende desde una perspectiva holística y de integración, a partir de la interrelación que se establece entre las partes que la conforman como un todo coherente; esto permite una visión más compleja sobre la práctica archivística.

- La gestión documental se realiza a partir de procesos que ocurren de manera recursiva, en un continuo de espacio-tiempo, lo cual permite satisfacer las diversas necesidades de información en diferentes momentos.

- El modelo se presenta como un marco multidimensional que permite una mejor comprensión de los diferentes roles de los archiveros contemporáneos en el tratamiento documental, y que resulta más consistente que el ciclo de vida para gestionar documentos en cualquier soporte por su carácter dinámico, fluido, intemporal y complejo.

- Presenta un enfoque amplio del concepto de evidencia archivística, toda vez que abarca diferentes contextos (de negocio, jurídico, cultural, histórico, personal, social). Asimismo, entiende que el archivo incluye todo rastro de actividad social, cultural y organizativa.

- Desde la perspectiva del MCD se ha defendido un enfoque de procedencia que trasciende la visión tradicional que la enmarca dentro de una perspectiva única y en estrechos límites institucionales. En este sentido, el pensamiento de la continuidad comprende una procedencia que puede ser múltiple, simultánea y paralela, a partir del reconocimiento de la participación de varios agentes (cocreadores) en la creación documental, y de la importancia de tener en cuenta las voces que, por diversas razones, han sido excluidas de la casa de la memoria.

- De esta manera, los archiveros asumen un rol más coherente con las necesidades, expectativas e intereses de la sociedad contemporánea, y 
contribuyen activamente con la construcción de entornos de relaciones políticas respaldadas por el acceso a la memoria social, la rendición de cuentas, la transparencia, la participación y la justicia social.

- Condiciona una mirada crítica sobre la teoría, la investigación y la práctica archivística, lo que permite desarrollar estrategias integrales y acciones de transformación de la propia praxis, así como desde lo político y lo social. Sobre la base de este enfoque crítico, los individuos y la comunidad tienen la capacidad de participar y colaborar junto con los archiveros en los procesos de gestión documental y en la construcción de la memoria colectiva.

- El modelo enfatiza en la naturaleza probatoria, transaccional y contextual del documento, lo cual permite servir a múltiples propósitos (gobernanza, rendición de cuentas, construcción de la memoria, etc.).

Upward (1996: 276-277) declaró cuatro principios clave que representan la naturaleza del modelo en cuestión.

- Un concepto de documento que sea inclusivo de aquellos de valor continuado que bace énfasis en sus usos a efectos transaccionales, de evidencia y memoria y que unifica las aproximaciones de archivo y gestión documental, tanto si se conservan por una fracción de segundo o por un milenio. Este principio se basa en el valor continuado del documento y el rol que cumplen como traza, evidencia y memoria. Parte del rechazo al enfoque divisorio que separa la gestión documental de la administración de archivo.

- Un enfoque de los documentos como entidades lógicas más que físicas, con independencia de si están en forma de papel o electrónica. Desde este principio el MCD es aplicable a todo documento, independientemente de su formato (impreso o digital). Concibe al documento, no como un objeto físico, sino como una entidad cuyos componentes y atributos se manifiestan en forma lógica.

- La institucionalización del rol de la profesión de gestión documental requiere un énfasis en la necesidad de integrar la gestión documental en los procesos y propósitos de negocios y sociales. Upward (1996) parte de la consideración de que los documentos son capturados en el contexto de las acciones de las que forman parte y en las que se encuentran recursivamente implicados. Al ser así carece de sentido pensar en los sistemas archivísticos desligados de los procesos de negocios y sociales de los cuales los documentos son sus productos. 
- La archivística es el fundamento para organizar el conocimiento acerca de la gestión documental. Se reconoce a la archivística como la ciencia que rige el conocimiento y los fundamentos en torno a la gestión documental. Al mismo tiempo, Upward (1996) advierte la necesidad de identificar el conocimiento proveniente de otras disciplinas que sean relevantes para este campo.

Los primeros tres principios fueron utilizados para presentar la reflexión sobre el SNARC, el cual ha atravesado un camino lleno de aciertos y desaciertos.

\section{El Sistema Nacional de Archivos de la República de Cuba: ANTECEDENTES}

La historia de los archivos en Cuba, desde los inicios del periodo colonial en 1511 hasta 1959, estuvo marcada por una práctica archivística poco sistematizada debido a la carencia de políticas gubernamentales para regular el tratamiento de los fondos y el desarrollo de los archivos. A lo largo de la colonización española se destruyeron importantes volúmenes de documentos de manera indiscriminada, por la falta de un adecuado proceso de selección y valoración documental.

Durante el periodo que va desde el final de la guerra en 1898, pasando por la ocupación militar estadounidense e instauración de la república neocolonial, hasta 1959 (momento en que triunfa la Revolución cubana), se emitieron varias normas jurídicas sobre la actividad archivística. Sin embargo, no se establecieron disposiciones concretas para regular procesos tan sensibles como el de valoración documental, salvo la publicación de algunas normativas que regulaban la destrucción, el préstamo y la extracción de documentos del Archivo Nacional (López, León y Marchena, 2012).

En 1942 se aprueba la Ley 6 donde se declaran, en su artículo primero, los documentos de utilidad pública, considerados parte del patrimonio nacional. Se refería a aquellos documentos que estaban relacionados con la actividad de orden público, de interés nacional y, por tanto, de valor para la historia, para los que se prohibía su comercialización o destrucción. Además, establecía la construcción de una edificación con las condiciones de capacidad y seguridad adecuadas para el Archivo Nacional. Su reglamento sería aprobado en 1948 y abordaba, entre otros aspectos, el régimen y contenido de la institución, distribución de sus fondos, organización técnica y administrativa (Llaverías, 1949: 319). 
No es hasta 1960 que se aprobó la Ley 714. En esta disposición jurídica se establecieron aspectos importantes como el reconocimiento del papel rector del Archivo Nacional sobre el control, organización, conservación y custodia del patrimonio documental, al tiempo que se consideraba como institución depositaria de todos los documentos legales producidos por el Estado. También se estipulaba la obligatoriedad de transferir a este archivo la documentación de las dependencias de los poderes ejecutivos, legislativos y judiciales.

En 2001 se publicó el DL 221 "De los Archivos de la República de Cuba", a partir del cual ocurre un cambio sustancial respecto al tratamiento documental (en especial sobre el proceso de valoración). En primer lugar, se crea el Sistema Nacional de Archivos, constituido por el Archivo Nacional de la República de Cuba (ANARC), los archivos históricos, los centrales, los de gestión, los fondos de particulares que decidieran integrar sus documentos al sistema, y de otros que tuvieran en su poder materiales especiales. Conjuntamente, se creó la Comisión Nacional de Control y Peritaje, un órgano conformado por especialistas de los archivos históricos y de las instituciones involucradas en la depuración de documentos.

La publicación del precitado decreto ley marcó un salto sin precedentes en la práctica archivística cubana a partir de la creación de un sistema para el tratamiento de los archivos en el país y de una comisión nacional para autorizar la depuración de los diferentes archivos y controlar la labor de las comisiones de cada organismo. Otros elementos que incorpora son las definiciones de archivo y documento de archivo, ausentes en la disposición anterior. Además, se define el archivo privado y se reconoce el derecho a la propiedad privada.

Este decreto ley contó con un reglamento publicado en 2004: la Resolución 73 del Ministerio de Ciencia, Tecnología y Medio Ambiente. Para cuestiones más específicas el Archivo Nacional de la República de Cuba publicó el Manual de procedimientos para el tratamiento documental (Marchena et al., 2008), en el que se detallan las particularidades relacionadas con los procesos archivísticos y que se encuentra vigente en la actualidad.

En 2009 se emitió el DL 265, el cual deroga el anterior y "establece las disposiciones generales para la protección del Patrimonio Documental de la Nación, así como las normas y principios que rigen la Gestión Documental en el territorio nacional" (481). Esta es la principal normativa jurídica que rige la actividad archivística en la actualidad y no cuenta aún con un reglamento.

\section{El SNARC DESDE LOS ENFOQUeS DEL MCD}

Si se compara el primer principio del MCD planteado por Upward (1996) con el caso cubano, se observa una clara diferencia: el SNARC presenta un diseño 
desde el punto de vista estructural y funcional que responde a las etapas del ciclo de vida del documento, como se declara en el Manual de procedimientos para el tratamiento documental (Marchena et al., 2008). De tal manera, establece tres edades o etapas por las que atraviesan los documentos en correspondencia con su uso. Cada edad o etapa está relacionada con su guarda y custodia en un tipo de archivo (archivo de gestión, central o histórico).

Asimismo, el SNARC toma como referencia los valores primarios y secundarios de los documentos para su transferencia, acceso, conservación o eliminación de forma total o parcial. Con respecto al valor secundario, aclara que es "el que interesa a los investigadores sobre la información retrospectiva" (Marchena et al., 2008: 29). Tal noción responde a la concepción del norteamericano Schellenberg (1956), quien consideraba que para que los documentos (records) fueran considerados archivos (archives) debían ser conservados por razones distintas de aquellas por las que fueron creados o acumulados, o sea para ser usados para referencia e investigación. De esta manera se ha asentado, en gran parte de la comunidad archivística cubana, un enfoque sobre el documento como aquél al que se le ha reconocido un valor para la historia, excluyente de aquellos que se encuentran en sus contextos más inmediatos de creación.

Las consecuencias de esta perspectiva fragmentada y dicotómica sobre el documento, con una marcada concepción histórica y patrimonial, se aprecian desde el propio marco regulatorio del SNARC. Es por ello que Mayra Mena Mugica, profesora titular de la Universidad de La Habana, ha aseverado que existe un desbalance entre la Resolución 60/11 y el DL 265/09: la primera estructura el control interno a partir de la consideración de evidencias para la transparencia y la rendición de cuentas; sin embargo, el decreto ley se enfoca en la preservación de esas evidencias como fuentes para la investigación, no en el sentido de garantizar que la información que se crea y se mantiene en la organización tenga calidad para poder tomar decisiones, rendir cuentas y enfrentar procesos de fraude y corrupción (Paz Martín, 2018).

Esto tiene mucha relación con el hecho de que los archiveros cubanos se identifiquen como simples custodios, cuando autores como Gilliland (2014) han insistido en que este enfoque conservacionista y de custodia resulta absolutamente inoperante.

Por ello los archiveros cubanos, en correspondencia con los procesos de cambio que está experimentando la sociedad cubana y a tono con el desarrollo revolucionario de las tecnologías de información y comunicación a nivel internacional y su irrupción en los procesos organizacionales en el país, deben revindicar su rol como profesionales de información desde una concepción amplia e inclusiva, que reconozca la complejidad de la realidad archivística en la que se encuentran insertados. 
En este orden de ideas, el pensamiento archivístico australiano se ha sustentado en una reconciliación de los valores de evidencia y memoria del documento, como se ha hecho ver desde su propio MCD, cuyas dimensiones sólo pueden ser comprendidas en un ir y venir de relaciones múltiples, que acontecen de manera fluida, contrario al modelo lineal y secuenciado del ciclo de vida, que fragmenta el documento en dos partes que tienen lugar en momentos y lugares distintos. De igual modo ha unificado lo que desde el ciclo de vida se ha considerado como dos profesiones separadas y dos profesionales distintos: el gestor documental (relacionado con la evidencia de actos y transacciones) y el archivero (relacionado con documentos de valor histórico y patrimonial).

De no comprender que el documento, objeto de estudio de la archivística, es una entidad cuyo valor como evidencia y memoria no se encuentra disociado en el espacio y el tiempo, entonces no será posible asumir con objetividad los actuales desafíos a los que se enfrentan los archiveros cubanos. Esos desafíos, como son el descomedido volumen documental que enfrentan las organizaciones, unido a pérdidas incalculables del patrimonio documental, lo que provoca que las administraciones no tengan capacidad para rendir cuentas, ni ser transparentes, requieren una visión de integración entre la gestión documental y la administración de archivos.

Como ha mencionado McKemmish (2017: 137), "una práctica integrada de gestión documental y archivo se caracteriza por la forma de atestiguar y de hacer memoria con un papel fundamental en la gobernanza, la rendición de cuentas, la identidad, la memoria individual y colectiva, la justicia social y el patrimonio cultural".

El segundo principio de Upward (1996), que concibe a los documentos como entidades lógicas más que físicas, aunque incluye los documentos impresos, resulta esencial para el tratamiento de aquellos que se generan y mantienen formato digital. Estos presentan unas particularidades técnicas como la separación de sus dimensiones física y lógica, una estructura morfológica de la información binaria y una forma de almacenamiento distribuido, entre otras, que exigen un acercamiento distinto a la manera tradicional de gestión.

El actual modo de gestión por parte del SNARC se basa en una comprensión del documento como entidad física y tangible, como se puede apreciar en la Resolución 41/09, en la que la mayoría de los requisitos relacionados con los documentos en formato electrónicos se enfoca en los soportes tecnológicos. Esta mirada es coherente con la propia noción de documento que presenta el DL 265/09, cuya definición comienza por decir: "medio en el que se registra o por el que se transmite información [...]” (481). 
Upward (1996) hace referencia a las críticas de Bearman (1989) sobre los inconvenientes de las aproximaciones físicas en la profesión archivística, y es que, como hace ver Flynn (2001), los atributos que le otorgan a un documento sus cualidades esenciales como el contenido, la estructura y el contexto no están necesariamente presentes físicamente en ambientes electrónicos, aun cuando estos sean visibles. De igual manera, Cook (2001) sugirió que los documentos, en los nuevos entornos de gestión, dejan de ser objetos físicos para volverse una entidad conceptual, controlada por metadatos que combinan contenido, contexto y estructura para proporcionar evidencia de las funciones de su productor.

Esa pérdida de corporeidad ha trastocado las nociones sobre las que tradicionalmente los archiveros han desarrollado su práctica profesional, como la procedencia y la custodia. Respecto a este último aspecto, el SNARC enfrenta una problemática en cuanto a la gestión de documentos digitales, a partir de los enfoques teóricos que sustentan la práctica profesional de sus archiveros, desde una perspectiva custodial.

El modelo de custodia física imperante en el SNARC, basado en un esquema de transferencia de responsabilidades según las etapas del ciclo de vida, ha condicionado cierta incapacidad para desarrollar una gestión documental estratégica y proactiva, como se requiere para tratar de manera efectiva los documentos digitales.

La creciente producción documental digital que se genera en las organizaciones y la falta de una perspectiva de intervención temprana sobre ellos por parte de los archiveros, que se mantienen anclados a una custodia física sobre objetos físicos, está afectando cada vez más la capacidad de esas organizaciones para preservarlos y mantenerlos a mediano y largo plazo. En la mayoría de los casos los productores se encuentran imposibilitados de transferir sus documentos digitales hacia una custodia archivística por razones administrativas, financieras y legales, aun cuando tengan un valor histórico y cultural.

Por otro lado, buena parte de los archivos centrales e históricos cubanos carecen de condiciones tecnológicas para recibir transferencias de documentos digitales y mantenerlos usables. De esa manera, los documentos que se generan digitalmente quedan fuera de los controles del sistema archivístico. Lo que ocurre, y esto es una preocupación en Cuba, es que este tipo de documentos se elimina de manera discrecional, según el criterio de cada productor.

Lo primero a comprender es que los archiveros cubanos hoy están tratando con un tipo documental cuyas características exigen una intervención desde antes de la creación, porque, entre otras razones, los sistemas necesitan tener incorporados de antemano los requisitos que son necesarios para 
garantizar la confiabilidad, autenticidad, integridad y usabilidad a lo largo del tiempo.

Es por ello que Bearman $(1989 ; 1996)$ ha insistido en la necesidad de que los archiveros intervengan de manera temprana en el proceso de gestión documental, incluso antes de la creación, o sea, en la etapa de diseño de sistema (Bantin, 1998). Como aseveró Cumming (2010), a su vez, la preservación y el mantenimiento del significado, usabilidad y carácter de evidencia de los documentos depende de cómo estos se crean y usan.

El MCD ofrece una visión de los documentos como entidades conceptuales, multicapas, que se interrelacionan con múltiples contextos y cuyos elementos de calidad, como la autenticidad, la confiabilidad, la integridad, entre otros, no pueden ser garantizados por medio de la custodia física y una práctica retroactiva, sino con un modo de actuación proactivo, que se sustente en la implementación de requisitos funcionales, la gestión de metadatos, el diseño y la aplicación de políticas archivísticas.

Para analizar el SNARC desde la perspectiva del tercer principio de Upward (1996), asociado a la institucionalización del rol de la profesión de gestión documental, debe tomarse en consideración que ya antes se había señalado la falta de correspondencia entre el DL 265/09 y otras normativas del marco regulatorio cubano como la Resolución 60/11. Ciertamente, el precitado decreto ley presenta una marcada inclinación patrimonial, en detrimento de un enfoque más operativo y de integración en los procesos organizacionales, como hoy se demanda en otras regulaciones.

Aspectos como transparencia y rendición de cuentas y responsabilidad administrativa no son tratados en el marco regulatorio archivístico, ni en el Manual de procedimientos para el tratamiento documental (Marchena et al., 2008). Aunque se define la gestión documental, esta categoría no se desarrolla de forma alguna, por lo que no queda claro cómo ha de intervenir la gestión documental y los archivos en general en los aspectos antes mencionados, como si el sistema archivístico estuviera ajeno a las necesidades y demandas de los propios productores.

Cuba cuenta con un conjunto de normas jurídicas que repercuten en la gestión documental de manera directa. El Reglamento de la Ley 107/11 plantea un grupo de deberes, entre los que se encuentra establecer sistemas que garanticen la confiabilidad y oportunidad de la información. La Resolución 60/11 establece, en su componente Información y Comunicación, la necesidad de que las organizaciones dispongan de información oportuna y fiable, así como de definir sistemas de información para el sustento de las actividades operativas, financieras y las relacionadas con los objetivos, metas y estrategias organizacionales. También insiste en la rendición transparente de cuentas sobre el uso de los bienes y recursos públicos. 
De igual modo, el DL 281/11 hace referencia de forma explícita a la gestión documental y establece un conjunto de principios que apuntan a la relevancia, oportunidad y veracidad de la información, la responsabilidad de los funcionarios respecto a la información relevante, la integración armónica de los componentes que integran el Sistema de Información del Gobierno, entre otros. Sobre la base de estas ideas, expone un conjunto de procesos como generación, procesamiento, almacenamiento, uso, descarte, etc., que considera clave para que la administración se gestione de manera transparente y responsable, así como para garantizar un ambiente de control. En el contexto empresarial el DL 281/07 insiste en que la información debe ser confiable, auténtica e íntegra, así como garantizar su adecuada custodia y almacenamiento.

Podrían mencionarse otros ejemplos como la propia Constitución de la República, que establece requisitos que inciden en la gestión documental en las organizaciones cubanas, pero sería extenderse demasiado en este punto. Lo cierto es que existe una necesidad reconocida de implementar sistemas y procesos de gestión documental en la gestión organizacional para sustentar la eficacia en la toma de decisiones, el control interno, la gestión de riesgos, la transparencia administrativa, la rendición de cuentas y un entorno de trabajo más democrático y responsable. Sin embargo, el SNARC y su marco regulatorio parecieran desligados de esa realidad, enmarcados de manera exclusiva en la protección del patrimonio documental.

Esto ha contribuido a que los decisores y funcionarios públicos cubanos muestren cierta falta de percepción respecto de la importancia y utilidad de los sistemas archivísticos en su quehacer diario, como se ha podido constatar desde algunos diagnósticos realizados por la Facultad de Comunicación de la Universidad de La Habana (Llano, 2011; Díaz, 2016; Aldao, 2016).

Por otro lado, los sistemas archivísticos, en correspondencia con los cambios en el modelo cubano de desarrollo económico y social concebido hasta 2030, necesitan una mayor orientación de sus procesos hacia los intereses y valores de la sociedad, contrario a la concepción sobre la que se sustenta hoy el SNARC.

La Conceptualización del Modelo Económico y Social Cubano de Desarrollo Socialista, aprobada en el VII Congreso del Partido Comunista de Cuba, establece en su capítulo tres "La Dirección Planificada de la Economía", el fomento de "la participación de los ciudadanos, la transparencia, el escrutinio público y la rendición de cuentas" (Partido Comunista de Cuba, 2016: 13). Tales propósitos son incompatibles con una práctica que se subordine a los intereses de la investigación histórica y del Estado, como ha defendido Cook (1997) en referencia al proceso de valoración, pues como manifestó Booms (1987: 106) "el público como un elemento constitutivo de la sociedad moderna [...] genera el proceso socio-político y legitima la autoridad política". 
Así, un elemento que necesariamente viene a colación es el acceso, cuya centralidad para los sistemas archivísticos se justifica en la necesidad de garantizar entornos más democráticos y participativos. En el caso cubano, se trata de un tema todavía pendiente. Por ejemplo, el Manual de procedimientos para el tratamiento documental (Marchena et al., 2008) del SNARC especifica que la documentación que se transfiere hacia los archivos históricos es de libre acceso a excepción de "[...] documentos que afecten a materias clasificadas de acuerdo con la Ley de Secretos Oficiales, o cuya difusión pueda entrañar riesgos para la seguridad y defensa del Estado" y "[...] aquellos documentos que contengan datos de carácter personal, cuyo conocimiento por terceros pueda suponer una injerencia en el honor o la intimidad de las personas" (Marchena et al., 2008: 33).

En el propio DL 265/09 se establece, en el artículo 53, que aquellas instituciones que son fuente de completamiento y tienen en su poder información oficial, limitada o clasificada, deben conservarla hasta que pierdan esa condición, según el DL 199/99, para entonces ser transferida al archivo que corresponda donde se definen restricciones de acceso, en los casos que sean necesarios (DL 265/09).

Ciertamente, estas normas jurídicas (DL 265/09 y DL 199/99) no aclaran el tiempo de vencimiento de tal condición (se refiere a la condición de clasificada), lo cual permite la restricción del acceso a la documentación que se encuentre en esa categoría de forma indefinida, con lo cual queda el acceso en un marco de decisión discrecional, aspecto que resulta contradictorio con respecto a los propósitos de transparencia y rendición de cuentas que se establecen en la Resolución 60/11.

Si la custodia ha sido el elemento distintivo de la archivística más tradicional, el acceso es uno de los más significativos en el nuevo paradigma, en tanto el uso es lo único que justifica la existencia de los archivos (Menne-Haritz, 2001). En este sentido, se hace necesaria una revisión crítica de la legislación archivística cubana, de manera que el SNARC pueda contribuir al desarrollo de una sociedad más abierta, participativa y democrática.

Asimismo, los archiveros cubanos necesitan abandonar su zona de confort para implicarse en cuestiones que van más allá de la conservación, la restauración y la custodia del patrimonio documental, para involucrarse en las actuales dinámicas organizacionales y sociales del país.

\section{A MANERA DE CONCLUSIONES}

Cuba cuenta con una larga tradición archivística desde el periodo colonial en 1511. Sin embargo, no es sino hasta 2001 que se constituye un Sistema 
Nacional de Archivos a partir de la publicación del DL 221/01. Desde entonces, el sistema se ha sustentado sobre la base de los enfoques y modelos teóricos de Europa Occidental y del norte de América.

La marcada orientación custodial y patrimonial de la práctica archivística cubana, así como su apego irrestricto a los tradicionales enfoques basados en el ciclo de vida y las teorías de Schellenberg, como se advierte en su marco regulatorio actual (DL 265/09, Resolución 41/09 y el Manual de procedimientos para el tratamiento documental (Marchena et al., 2008) del SNARC), han presupuesto algunas debilidades que a juicio de estos autores requieren atención inmediata por las autoridades archivísticas del país:

- Inobservancia por parte del SNARC de las necesidades informacionales de los productores de documentos (organizaciones de la administración pública y el sector empresarial cubano) por falta de una visión integradora y de complejidad sobre la realidad archivística cubana que envuelve aspectos como la seguridad de la información, el control interno, la calidad de los servicios, la confianza pública, la eficacia en la toma de decisiones a partir de información confiable, auténtica, íntegra, usable, accesible. Todo ello guarda una estrecha relación con el hecho de que los archiveros cubanos comprendan que su objeto de trabajo no se limita al documento que ha llegado a una institución archivística, sino a cualquier ítem de evidencia de valor continuado.

- Incapacidad por parte de los archiveros cubanos para desarrollar una gestión documental proactiva y estratégica, como hoy la sociedad cubana demanda ante la creciente producción de información que se genera en diversos formatos y que ha provocado una especie de infoxicación en las organizaciones, tanto como pérdida, manipulación y eliminación descontrolada de documentos. Ese escenario afecta invariablemente la capacidad de las organizaciones para rendir cuentas sobre sus actos con transparencia, sobre la base de un ambiente de confianza. Para enfrentar esta problemática los archiveros necesitan involucrarse en los procesos documentales desde las etapas de diseño de sistemas sobre los que se gestionarán los documentos. Así, la creación, captura, organización y acceso deberán ser procesos que se llevan a cabo como parte de una realidad continua de la que los archiveros sean parte de forma natural.

- Ineficacia del SNARC para gestionar documentos en formato digital de manera efectiva. Cómo preservar los documentos que nacen en ambiente digital es una de las preocupaciones de los archiveros en Cuba. En las organizaciones tanto de la administración pública como 
del sector empresarial, han experimentado un incremento exponencial de la información electrónica en diferentes formatos, por lo que un volumen importante del patrimonio documental de la nación cubana en la actualidad se encuentra en ambiente no tradicional. Esta documentación precisa intervención archivística desde la creación y la captura.

Un acercamiento al pensamiento del continuo, desde los principios declarados por Frank Upward, implicaría necesariamente una concepción de integración al interior del SNARC, que se desmarque de una práctica sustentada en la custodia física y la transferencia de responsabilidades, según etapas que suceden en el tiempo y que fragmentan una realidad que requiere ser comprendida y tratada desde su complejidad.

La visión de continuidad implica, por tanto, un cambio en el tradicional rol de los archiveros cubanos para comenzar a involucrarse en el análisis y diseño de sistemas, gestión de metadatos, auditoría de gestión documental, evaluación de servicios, entre otros procesos. Para ello, se hace necesario revisar el marco regulatorio archivístico cubano, en aras de acercarlo a las necesidades y demandas de la sociedad cubana actual y a los desafíos que las organizaciones cubanas ya vienen enfrentando.

Los autores de este artículo reconocen en el MCD, concebido por Frank Upward y sus colegas de la Universidad de Monash, un marco de buenas prácticas que podría fortalecer el SNARC y contribuir con una gestión más eficaz en el complejo entorno en el que la sociedad cubana se descubre.

\section{REFERENCIAS}

Aldao, Janet. 2016. "Diagnóstico de la Gestión Documental en los Órganos de la Administración Central del Estado Cubano”. Tesis de licenciatura, Universidad de La Habana, Facultad de Comunicación.

Bantin, Philip. 1998. "Strategies for Managing Electronic Records: A New Archival Paradigm? An Affirmation of our Archival Tradition”. Archival Issues 23: 15-34.

Bearman, David. 1989. Electronic evidence: strategies for managing records in contemporary organizations. Pittsburgh: Archives \& Museum Informatics.

Bearman, David. 1996. "Item Level Control and Electronic Recordkeeping. Archives and Museum Informatics". Cultural Heritage Informatics Quarterly 10 (3): 242-245.

Booms, Hans. 1987. "Society and the formation of a documentary heritage: issues in the appraisal of archival sources”. Archivaria 24: 69-107. 
Cook, Terry. 1997. "What is Past is Prologue: A History of Archival Ideas Since 1898, and the Future Paradigm Shift". Archivaria 43: 17-63.

Cook, Terry. 2001. "Archival science and postmodernism: new formulations for old concepts". Archival Science (1): 3-24.

Cumming, Kate. 2010. "Ways of seeing: contextualizing the continuum”. Records Management Journal 20 (1): 41-52.

Cunningham, Adrian. 2012. "Peter J. Scott and the australian 'series'system: main features and historical context", en Peter J. Scott and the australian 'series' system: its origins, features, rationale, impact and continuing relevance, editado por Adrian Cunningham, Laura Millar y Barbara Reed, 2-10. International Congress on Archives, Australia, agosto de 2012.

Decreto Ley 199. 1999. "Sobre la Seguridad y Protección de la Información Oficial". Gaceta Oficial. Cuba: Consejo de Estado.

Decreto Ley 221. 2001. "De los Archivos de la República de Cuba”. Gaceta Oficial. Cuba.

Decreto Ley 265. 2009. "Del Sistema Nacional de Archivos de la República de Cuba”. Gaceta Oficial. Cuba.

Decreto Ley 281. 2007. "Reglamento para la Implantación y Consolidación del Sistema de Dirección y Gestión Empresarial Estatal”. Gaceta Oficial. Cuba.

Decreto Ley 281. 2011. "Del Sistema de Información del Gobierno". Gaceta Oficial. Cuba.

Díaz, Mirelys. 2016. "Diagnóstico del estado de la gestión documental en la Universidad de La Habana”. Tesis de maestría, Universidad de La Habana, Facultad de Comunicación.

Evans, Joanne, Sue McKemmish y Greg Rolan. 2017. "Critical Approaches to Archiving and Recordkeeping in the Continuum". Journal of Critical Library and Information Studies 1 (2): 1-38.

Flynn, Sarah J. A. 2001. "The Records Continuum Model in Context and its Implications for Archival Practice". Journal of the Society of Archivists 22 (1): 79-93.

Gilliland, Anne. 2014. "Reconceptualizing records, the archive and archival roles and requirements in a networked society". Knygotyra 63: 17- 34.

Kuhn, Thomas Samuel. 1971. "La estructura de las revoluciones cientificas". Ciudad de México: Fondo de Cultura Económica.

Ley 6. 1942. "De los Documentos que integran el Patrimonio Nacional y Resultan de Utilidad Pública”. Gaceta Oficial. Cuba.

Ley 714. 1960. "Del funcionamiento Orgánico del Archivo Nacional”. Gaceta Oficial. Cuba.

Llano, María. 2011. "La normalización de la gestión documental y su expresión en las regulaciones cubanas especialmente para documentos electrónicos”. Tesis de Maestría, Universidad de La Habana, Facultad de Comunicación.

Llaverías, Joaquín. 1949. Historia de los archivos de Cuba. 2a. ed. La Habana: Publicaciones del Archivo Nacional de Cuba.

López, Yorlys, Marisol León y Martha Marina Marchena. 2012. "Aspectos técnicos-jurídicos de la valoración documental. Un análisis a partir de la legislación archivística cubana". Boletín del Archivo Nacional, 18: 62-75. 
Marchena, Martha Marina, Olga María Valdés, Marisol León y Mercedes Llovet. 2008. Manual de procedimientos para el tratamiento documental. La Habana: Archivo Nacional de la República de Cuba.

McKemmish, Sue. 1997. "Yesterday, Today and Tomorrow: a continuum of responsibility". Trabajo publicado en Proceedings of the Records Management Association of Australia 14th National Convention. https://figshare.com/articles/Yesterday_ today_and_tomorrow_a_continuum_of_responsibility/4037433https://figshare. com/articles/Yesterday_today_and_tomorrow_a_continuum_of_responsibility/4037433

McKemmish, Sue. 2017. "Recordkeeping in the continuum: an australian tradition", en Research in the Archival Multiverse, editado por Anne Gilliland, Sue McKemmish, y Andrew J. Lau, 122-160. Australia:Monash University Publishing.

Menne-Haritz, Angelika. 2001. "Access - the reformulation of an archival paradigm”. Archival Science 1:57-82.

Partido Comunista de Cuba. 2016. "Proyecto de Conceptualización del Modelo Económico y Social Cubano de Desarrollo Socialista”. Proyecto aprobado en el 7mo Congreso del Partido Comunista de Cuba.

Paz Martín, Sarah. 2018. "Los riesgos del olvido. Entrevista a Mayra Mena Mujica, profesora de la Facultad de Comunicación de la Universidad de La Habana”. Cubahora. http://www.cubahora.cu/sociedad/los-riesgos-del-olvido-ii-y-final-ilustraciones

Piggott, Michael y Sue McKemmish. 2002. "Recordkeeping, Reconciliation and Political Reality”. Trabajo presentado en la Australian Society of Archivists Annual Conference, Sydney, agosto de 2012 http://staging-infotech.monash.edu.au/research/groups/rcrg/publications/piggottmckemmish2002.pdf

Reed, Barbara. 2012. "Standing on the shoulders of giants': the legacy of Peter Scott'sarchival thinking", en Peter J. Scott and the australian 'series' system: its origins, features, rationale, impact and continuing relevance, editado por Adrian Cunningham, Laura Millar, y Barbara Reed, 20-27. International Congress on Archives, Australia, agosto de 2012.

Reglamento de la Ley 107 "De la Contraloría General de la República de Cuba". 2011. Gaceta Oficial. Cuba.

Resolución 41. 2009. "Lineamientos para la Conservación de Fuentes Documentales”. Gaceta Oficial. Cuba: Ministerio de Ciencia, Tecnología y Medio Ambiente.

Resolución 60. 2011. "Sobre las Normas del Sistema de Control Interno". Gaceta Oficial. Cuba: Contraloría General de la República de Cuba.

Resolución 73. 2004. "Reglamento del Decreto Ley 221 'De los Archivos de la República de Cuba"'. Gaceta Oficial. Cuba.

Ribeiro, Fernanda. 2017. "La era poscustodial: implicaciones en el campo de la ciencia de la información", en La Archivística y la Ciencia de la Información Documental: autonomía e interdependencias, editado por Miguel Ángel Rendón Rojas, 23-37. Ciudad de México: Instituto de Investigaciones Bibliotecológicas y de la Información, UNAM.

Schellenberg, Theodore R. 1956. Modern archives: principles and techniques, 2a. ed. Chicago: University of Chicago.

Silva, Armando Malheiro y Fernanda Ribeiro. 2010. Paradigmas serviços e mediações em Ciência da Informação. Recife, Brasil: Nectar. 
Upward, Frank. 1996. "Structuring the records continuum - part one: Postcustodial principles and properties”. Archives and Manuscripts 24 (2): 268-285.

Upward, Frank. 1997. "Structuring the Records Continuum, Part Two: Structuration Theory and Recordkeeping". Archives and Manuscripts 25 (1): 10-35.

Upward, Frank. 2017. "The archival multiverse and Eddies in the Spacetime Continuum”,. en Research in the Archival Multiverse, editado por Anne Gilliland, Sue McKemmish, y Andrew J. Lau, 198-227. Australia: Monash University Publishing.

Para citar este texto:

Castillo Guevara, Jorge del y Sarah Paz Martín. 2019. “Reflexiones generales sobre el Sistema Nacional de Archivos de la República de Cuba desde la perspectiva del modelo de la continuidad de los documentos". Investigación Bibliotecológica: archivonomía, bibliotecología e información 33 (81): 89-110.

http://dx.doi.org/10.22201/iibi.24488321xe.2019.81.58033 